\title{
Article
}

\section{The Cross-sectional Shape and Height Expansion of Coronal Loops: High- resolution Coronal Imager (Hi-C) Analysis of AR 12712}

Williams, Thomas, Walsh, Robert William and Morgan, Huw

Available at https://clok.uclan.ac.uk/39306/

Williams, Thomas orcid iconORCID: 0000-0002-2006-6096, Walsh, Robert William orcid iconORCID: 0000-0002-1025-9863 and Morgan, Huw (2021) The Cross-sectional Shape and Height Expansion of Coronal Loops: High-resolution Coronal Imager (Hi-C) Analysis of AR 12712. The Astrophysical Journal, 919 (1). p. 47. ISSN 1538-4357

It is advisable to refer to the publisher's version if you intend to cite from the work. http://dx.doi.org/10.3847/1538-4357/ac0f76

For more information about UCLan's research in this area go to http://www.uclan.ac.uk/researchgroups/ and search for <name of research Group>.

For information about Research generally at UCLan please go to http://www.uclan.ac.uk/research/

All outputs in CLoK are protected by Intellectual Property Rights law, including Copyright law. Copyright, IPR and Moral Rights for the works on this site are retained by the individual authors and/or other copyright owners. Terms and conditions for use of this material are defined in the policies page. 


\title{
The Cross-sectional Shape and Height Expansion of Coronal Loops: High-resolution Coronal Imager (Hi-C) Analysis of AR 12712
}

\author{
Thomas Williams ${ }^{1,2}$ (D), Robert W. Walsh ${ }^{1}$ (D), and Huw Morgan ${ }^{2}$ (D) \\ ${ }^{1}$ Jeremiah Horrocks Institute, UCLan, Preston, PR1 2HE, UK
${ }^{2}$ Department of Physics, Aberystwyth University, Penglais, Aberystwyth, SY23 3BZ, UK \\ Received 2021 June 18; revised 2021 June 24; accepted 2021 June 27; published 2021 September 22
}

\begin{abstract}
Coronal loop observations have existed for many decades yet the precise shape of these fundamental coronal structures is still widely debated since the discovery that they appear to undergo negligible expansion between their footpoints and apex. In this work a selection of eight EUV loops and their 22 sub-element strands are studied from the second successful flight of NASA's High-resolution Coronal Imager (Hi-C 2.1). Four of the loops correspond to open fan structures with the other four considered to be magnetically closed loops. Width analysis is performed on the loops and their sub-resolution strands using our method of fitting multiple Gaussian profiles to crosssectional intensity slices. It is found that while the magnetically closed loops and their sub-element strands do not expand along their observable length, open fan structures may expand an additional 150\% of their initial width. Following recent work, the Pearson correlation coefficient between peak intensity and loop/strand width are found to be predominantly positively correlated for the loops $(\approx 88 \%)$ and their sub-element strands $(\approx 80 \%)$. These results align with the hypothesis of Klimchuk \& DeForest that loops and-for the first time-their sub-element strands have approximately circular cross-sectional profiles.
\end{abstract}

Unified Astronomy Thesaurus concepts: Solar coronal loops (1485); Solar corona (1483); Solar active regions (1974)

\section{Introduction}

It has long been shown that coronal loops may have uniform cross sectional widths along their observable length (Klimchuk et al. 1992; Klimchuk 2000; Watko \& Klimchuk 2000) where the cross-sectional profile is roughly circular. This behavior is contrary to what one would expect; that is, flux tubes, and thus coronal loops are expected to expand with height to maintain the pressure balance between the internal and external loop plasma due to gravitational stratification and decreasing magnetic field strength. This perceived circular cross section may be explained by locally twisted flux tubes (Klimchuk et al. 2000; López Fuentes \& Klimchuk 2006), which have been demonstrated to undergo less expansion than untwisted structures (McClymont \& Mikic 1994).

The findings of Malanushenko \& Schrijver (2013) offer an explanation for the perceived lack of expansion in loop observations that one would expect-the loops may have noncircular cross sections such as an ellipse. As noted by Klimchuk \& DeForest (2020), if the expansion occurs preferentially along the line of sight, i.e., radially, rather than in the image plane, then no contradiction occurs. Malanushenko \& Schrijver (2013) propose that because the coronal plasma is optically thin, loops expanding along the line of sight will be brighter in the image plane, which causes a selection bias as the brightness will decrease less rapidly as a function of height due to the increasing line of sight depth toward the apex. While it is possible that the cross-sectional aspect ratio may change along a loop's length, it is also possible that the aspect ratio remains fixed and merely rotates around a central axis, such as a twisting ribbon/rubber band (McCarthy et al. 2021). However, if that

Original content from this work may be used under the terms of the Creative Commons Attribution 4.0 licence. Any further distribution of this work must maintain attribution to the author(s) and the title of the work, journal citation and DOI. were the case then a loop would experience localized brightening and dimming along its length. That is, if we consider the twisting of an oval cross section, then there is more (less) material along the line of sight when the semiminor (major) cross-sectional axis is lined preferentially with the observer, which leads to brighter (dimmer) intensity in the 2D projection of observational data due to the increased (decreased) plasma along the line of sight.

To test this hypothesis, Klimchuk \& DeForest (2020) analyzed the relationship between the peak intensity and crosssectional width of 20 coronal loops within the $193 \AA$ High Resolution Coronal Imager (Hi-C; Kobayashi et al. 2014) data set whose lengths are $<60 \mathrm{Mm}$. They find that these two properties were either positively correlated or uncorrelated but not negatively correlated, suggesting the cross sections of the loops could be approximately circular. More recently, McCarthy et al. (2021) utilized observations from two vantage points with $171 \AA$ data from both the Solar Dynamics Observatory's Atmospheric Imaging Assembly (AIA; Lemen et al. 2012) and Solar Terrestrial Relation Observatory's Extreme Ultraviolet Imager (EUVI; Howard et al. 2008). From their sample of 151 loops, they identified 13 as being suitable for width analysis. Utilizing diameter-diameter measurements and peak intensity versus loop width graphs, McCarthy et al. (2021) deduce that four of the 13 loops may be elliptical with the remaining nine loops being approximately circular in shape. However, the diameter-diameter measurements are unlikely to be reliable given the lower resolution of EUVI, especially when AIA has been shown to be incapable of resolving-or in some instances detecting - narrower loops (Williams et al. 2020a).

As is discussed in Klimchuk (2000), the lack of cross-sectional expansion in loop observations have important implications for coronal heating models. The first is that energy may be deposited in an axial symmetric manner at the same spatial scales as the diameter of monolithic coronal loops/strands (FWHM $\lesssim 200 \mathrm{~km}$; 

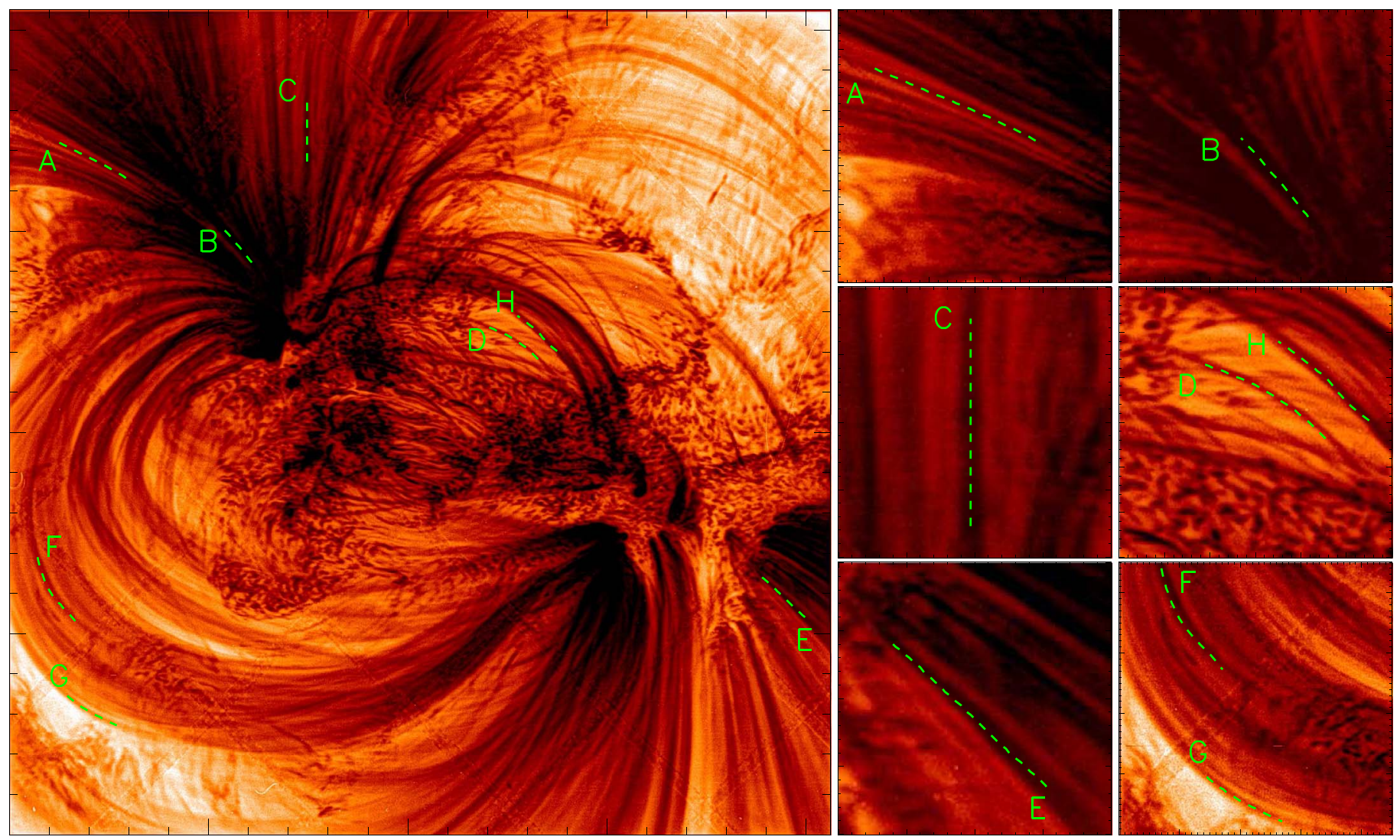

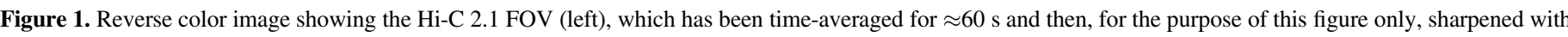

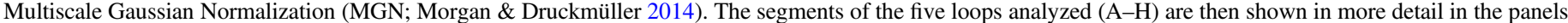
on the right.

Williams et al. 2020a, 2020b). Alternatively, the energy deposited within strands and loops occur at spatial scales much smaller than the diameters of these structures-perhaps on spatial scales as small as $15 \mathrm{~km}$ (Peter et al. 2013) —and is then transported orthogonal to the loop abscissa in an axisymmetric manner. One popular mechanism for the latter scenario is the nanoflare heating model (Parker 1983, 1988) whereby field lines become braided and undergo small-scale magnetic reconnection due to photospheric motion at the footpoints.

In light of recent $\mathrm{Hi}-\mathrm{C}$ findings indicating that monolithic loops may have approximately circular cross sections that exhibit no expansion along their observable length (Klimchuk \& DeForest 2020), this paper employs the methods used in previous studies (Section 2) to examine the spatial scales along the loop envelope (Williams et al. 2020a) and individual loop sub-element strands (Williams et al. 2020b) of eight distinct coronal structures. In Section 3, the relationship between loop width and their peak intensities are explored to classify whether their cross sections may be approximately circular (Klimchuk \& DeForest 2020) or oval such as a twisted ribbon (Malanushenko \& Schrijver 2013; McCarthy et al. 2021). For the first time, this study also extends this analysis to the sub-element strands contained within coronal loops and a discussion of these results in the context of current literature and the authors' concluding remarks are presented in Section 4.

\section{Data Preparation and Analysis Method}

On 2018 May 29 at 18:54 UT, Hi-C 2.1 was successfully relaunched from the White Sands Missile Range, NM, USA, capturing high-resolution data $(2 \mathrm{k} \times 2 \mathrm{k}$ pixels; $4.4 \times 4$ !. 4 field of view (FOV)) of target active region AR 12712 in EUV emission of wavelength $172 \AA$ (dominated by Fe IX emission $\approx 0.8 \mathrm{MK}$ ) with a plate scale of $0.129^{\prime \prime}$. During the flight Hi-C 2.1 captured 78 images with a $2 \mathrm{~s}$ exposure time and a $4.4 \mathrm{~s}$ cadence between 18:56 and 19:02 UT. Full details on the Hi-C 2.1 instrument can be found in Rachmeler et al. (2019).

\subsection{Data-set Extraction and Background Subtraction}

The basis of the sample data set investigated here include a number of subsets from the 10 higher-emission cross-section slices analyzed by Williams et al. (2020a) plus nine other additional slices from within the Hi-C 2.1 FOV (see Figure 1 where all data set locations are indicated). In each case the resulting emission profile across the structures would indicate substructure strands that are not fully resolved.

Following the method outlined in (Williams et al. 2020a), the Hi-C 2.1 data set under consideration is time-averaged over a period $\approx 60 \mathrm{~s}$ that is free from spacecraft jitter. ${ }^{3}$ Each cross section normal to each strand is taken to be 3 pixels deep and the background emission is then subtracted. As outlined in Figure 2 of Williams et al. (2020b), this background subtraction is performed by first finding all the local minima of a slice, and interpolating through these values using a cubic spline (Yi et al. 2015) to obtain a global trend (blue dashed line). The global

\footnotetext{
3 A consequence of the instability experienced during the Hi-C 2.1 flight is that ghosting of the mesh could not be avoided (Rachmeler et al. 2019). This leads to the diamond patterns across the entire Hi-C 2.1 FOV, which are exaggerated when the data is enhanced with MGN (Figure 1).
} 
trend is then subtracted from the intensity profile along the slice, leaving behind the background subtracted coronal strands (similar to Aschwanden \& Schrijver 2011; Williams et al. 2020a). Due to the large number of counts detected by Hi-C 2.1 the Poisson error associated with these isolated coronal strands is minimal.

\subsection{Gaussian Fitting and FWHM Measurements}

The analysis method is based on the assumption that at rest, an isolated coronal strand element has an observed emission profile across its width and normal to the strand axis that is approximately Gaussian. It is important to note that as indicated by Pontin et al. (2017), instantaneously coronal strands may not necessarily have a clear Gaussian cross section. On the other hand, (Klimchuk \& DeForest 2020) have shown from Hi-C observations that coronal strands are likely to have Gaussian cross sections. The data samples are time-averaged over $\approx 60 \mathrm{~s}$ (the first $11 \mathrm{Hi}-\mathrm{C} 2.1$ frames) to average out variations over short timescales, which helps address this issue. While no obvious signs of motion within the structures analyzed are noticed in this $60 \mathrm{~s}$ window, the authors acknowledge that as indicated by Morton \& McLaughlin (2013), small amplitude oscillations could be present which would lead to the measured widths being broader than the structural width due to the time integration performed.

The observed Hi-C 2.1 intensity profile of a cross-sectional slice is reproduced by simultaneously fitting Gaussian profiles, the number of which is determined by the Akaike information criterion (AIC; Akaike 1974) along with a corrective term (AICc) for small sample sizes. This is fully described in the appendix of Williams et al. (2020b). Subsequently, the FWHM of the Gaussian profile is measured to provide an estimate of the possible width of the substructures likely present within the Hi-C 2.1 data.

Thus, the method employed to fit Gaussian profiles to the observed Hi-C 2.1 intensity is as follows. First, the following expression for a Gaussian function, $Y_{G}$ is used:

$$
Y_{G}=A \exp \left(\frac{-\left(x-x_{p}\right)^{2}}{2 W^{2}}\right),
$$

where by $x$ is position along the cross-section slice, $A$ and $x_{p}$ are the amplitude and location of the peak, and $W$ is the Gaussian rms width. This can be related to the FWHM by FWHM = $2 \sqrt{2 \ln 2} W \approx 2.35 W$.

An estimate is made on the number of structures, $N$ that could be present within the intensity profile along with their approximate location, width, and amplitude. Summing the $Y_{G}$ values for $N$ number of Gaussian curves at each pixel yields the model fit:

$$
f(x)=\sum_{i=1}^{N} Y_{G(i)}(x) .
$$

The closeness of the fit at each pixel, $\chi^{2}(x)$ is then determined by measuring the deviation of the fit from the original intensity:

$$
\chi^{2}(x)=\left(\frac{f(x)-y(x)}{\sigma(x)}\right)^{2},
$$

where $y(x)$ and $\sigma(x)$ are the observed Hi-C 2.1 intensity and Poisson error at each pixel. The overall closeness of fit is then taken as $\sum \chi^{2}(x)$, which is then reduced to its smallest value by simultaneously adjusting the free parameters $A, x_{p}$, and $W$ for the $N$ Gaussian curves in $f(x)$. The minimization of $\sum \chi^{2}(x)$ is performed by using the nonlinear least-squares curve fitting method, MPFIT ${ }^{4}$ (Markwardt 2009), which is based on the MINPACK-1 FORTRAN library (Moré 1978). The $1 \sigma$ uncertainties returned from MPFIT are only accurate if the shape of the likelihood surface is well approximated by a parabolic function. Whether fitting multiple Gaussian profiles to each slice satisfies this condition or not would require analysis beyond the scope of this study, however, the $1 \sigma$ uncertainties do provide a lower-bound of the FWHM errors.

To determine the appropriate number of Gaussian profiles, $N$, within a given slice, the AIC model selection is employed. This is done by first generating several candidate models, where the number of Gaussian curves differs in each model. The nonlinear least-squares curve fitting method is then employed for each candidate model and finally the AICc is then computed. The model with the smallest AICc value is then selected as the preferred model for that Hi-C 2.1 slice. Once the number of Gaussian profiles contained within a Hi-C 2.1 slice is determined, the strand width(s) are taken as the Gaussian FWHM value(s).

Klimchuk \& DeForest (2020) state that this type of analysis is sensitive to background subtraction, and as such we find the number of Gaussian profiles fitted to a Hi-C 2.1 slice can be influenced by unwanted features such as other loops crossing in front/behind the loop(s) or underlying moss regions. While concerted efforts are made to avoid these regions, this is not always possible, and these unwanted overlaps of coronal structures result in differing numbers of sub-element strands being fitted by the AICc method compared to slices either side of the overlaps. Consequently, as this study aims to measure the changes in structural width and emission measure not only along loop segments but also along sub-element strands that may reside within these structures, a consistent number of Gaussian profiles is required to be fitted for the analyzed portion of the loop(s). It is reasonable to assume the number of sub-element strands along a loop segment would be consistent and thus the cross-sectional slices where the number of Gaussian profiles determined by the AICc model selection do not match the modal value are likely to be contaminated with other coronal features along the line of sight. With this in mind, the sub-element strands are only analyzed for the crosssectional slices where the number of Gaussian profiles matches the modal value of Gaussian's for the whole loop segment in question. A consequence of this conservative approach to fitting sub-element strands is that it is not always possible to analyze the structures along the entire loop segment. However, measuring the width of the loop envelope has no such constraints, and the method employed in Williams et al. (2020a) is adopted with the improved background subtraction discussed in Williams et al. (2020b).

\section{Results and Analysis}

A total of eight loops are selected from the Hi-C 2.1 FOV (Figure 1) that are relatively free from emission of surrounding coronal structures. From the selection of Hi-C 2.1 loops chosen for this study, loops A, B, C, and E are from open fan regions,

\footnotetext{
4 MPFIT is freely available at: http://purl.com/net/mpfit.

5 Visual inspection of the AIA and EUVI full disk images support the expectation that these loops are fan structures and not large, closed loops.
} 
while loops D, F, G, and $\mathrm{H}$ are magnetically closed structures. In the analysis that follows, the orientation of loops D, F, and $\mathrm{G}$ are north to south (i.e., position 0 is at the top of the close-up images of Figure 1), whereas the other loops are oriented south to north such that the segments of the structures are traced away from their nearest footpoint. The AICc determined modal number of sub-element strands within loops A, D, E, G, and $\mathrm{H}$ is three, while loops $\mathrm{B}$ and $\mathrm{C}$ contain four strands, and loop $\mathrm{F}$ has only two.

As can be seen in the FOV image, selecting a segment of a coronal loop that is isolated from surrounding coronal structures and the ghosting of the CCD mesh cells is a nontrivial task due to the abundance of loop-like structures and the underlying moss in AR 12712. Focusing on the close-up images of loops A-H in Figure 1 reveals that even the best-case loop segments we have selected here are not completely isolated from other coronal features or the mesh cell ghosting. For example, it is not possible to examine a longer portion of loop A as the segment analyzed (denoted by the dashed line) is enclosed on both ends by the ghosting of a mesh cell. Similarly, despite loop D having a high contrast compared to the surrounding corona in the MGN-enhanced images (Morgan \& Druckmüller 2014), there are several structures that intersect the loop and thus affect the background subtraction.

\subsection{Width Analysis}

In Figures 2 and 3 the top panels labeled B1-B6 and H1H16 show the Hi-C 2.1 cross-sectional intensity (blue), the AICc determined best-fit ( $r e d$ ), and the Gaussian profiles (gray) that generate the fit. The intensity of these panels are normalized with respect to the maximum peak intensity of the loop segment being considered. The same figures for the other six loops are shown in the Appendix (Figures 6-11). In all these plots for loops $\mathrm{A}-\mathrm{H}$ it is possible to follow the individual strands from one cross-sectional slice to the next along the loop segments analyzed. As with previous results using this method (Williams et al. 2020b), the AICc determined fits closely match the observed Hi-C 2.1 cross-sectional profiles in most instances, with some minor exceptions such as slices A3 and F6. Here, it would be possible to reduce the deviance between the generated fits and the Hi-C 2.1 emission by adding further Gaussian profiles, however, in doing so the curve fitting model would no longer be supported by the AICc model selection method and would increase the likelihood of overfitting the data along the length of the loop segments analyzed.

The bottom panels of Figures 2 and 3 show the variation in loop (black) and strand (cyan, magenta, green, orange) widths as a function of loop position. The error bars for the loop width indicate a Hi-C 2.1 pixel width $(93.525 \mathrm{~km})$ whereas the error bars of the strands are the $1 \sigma$ errors returned from MPFIT. From the eight loops considered, six of them show no signs of significant expansion, whereas the two loops that do (B and E) are open fan structures and may be expected to expand. Curiously, $\mathrm{A}$ and $\mathrm{C}$ are also open fan structures yet their widths remain largely constant along the segments analyzed. It could be the case that these structures have undergone expansion closer to the footpoints as with loops B and E, however, this cannot be verified in this data set with our current methods.

Figure 4 shows the separation between the peak intensity of neighboring sub-element strands as a function of loop position for the loops A-H. Increasing loop position for loops A, B, C,
D, and H (E, F, and G) correspond to a south-to-north (northto-south) orientation in Figure 1. Focusing upon the six loops that show no obvious expansion, their sub-element strands also exhibit no expansion (Figures 3, 6, 7, 8, 10, 11) or separation between adjacent strands (Figure 4). Of these loops, only loops $\mathrm{C}$ and $\mathrm{F}$ show initial and final widths that are above the observational error of two Hi-C 2.1 pixel widths $(187.05 \mathrm{~km})$. For loop C, this level of expansion is insignificant $(80 \mathrm{~km}$ above $2 \mathrm{Hi}-\mathrm{C} 2.1$ pixels), and the close proximity of a loop to the east whose intensity increases when tracing the structure in a south-to-north orientation (Figure 1) may effect the background subtraction performed on loop $\mathrm{C}$, which would account for the decreased width seen from loop position $14 \mathrm{Mm}$ onward in Figure 7. For loop F, the width is predominantly the same along the loop segment until a sudden decrease is seen in the final three cross-sectional slices (Figure 10). As can be seen in the cross-sectional slices and from the visual reference in Figure 1, the loop intensity decreases relative to the surrounding loop bundle and thus the magnitude of the loop intensity relative to the background emission that is subtracted decreases, which may contribute to the contraction in loop width indicated in Table 1.

To quantify the total expansion $\left(\Gamma_{\text {tot }}\right)$ of each loop/strand, the final and initial widths of the segment analyzed are calculated as the ratio

$$
\Gamma_{\mathrm{tot}}=\frac{W_{\mathrm{final}}}{W_{\text {initial }}} .
$$

The expansion rate $\left(\Gamma_{\text {rate }}\right)$ is then simply expressed as

$$
\Gamma_{\text {rate }}=\frac{\Gamma_{\text {tot }}}{l},
$$

where $l$ is the length of the loop/strand segment.

Employing this on loops $\mathrm{B}$ and $\mathrm{E}$, it can be seen that they expand a total of $\approx 860 \mathrm{~km}$ and $\approx 980 \mathrm{~km}$, giving expansion rates $\left(\Gamma_{\text {rate }}\right)$ along their lengths of $4.2 \%$ and $6.8 \%$ per $\mathrm{Mm}$, respectively (Table 1). For Loop B, the widths of strands 1 and 2 remain within two pixel widths and as such do not contribute to the expansion of Loop B, while strands 3 and 4 expand $\approx 500 \mathrm{~km}$ and $\approx 320 \mathrm{~km}$. Furthermore, Figure 4 reveals that while the separation between strands 1 and 2 remains roughly constant, strands 2 and 3, and strands 3 and 4 spread apart along the loop segment under consideration. If we consider the findings of McClymont \& Mikic (1994) that a twisted structure exhibits less expansion than an untwisted structure, then it may be that strands 1 and 2 are twisted structures while strands 3 and 4 are subjected to less twist and thus expand with height.

For loop E, all three strands show expansion above two pixel widths ( $\approx 260 \mathrm{~km}, \approx 460 \mathrm{~km}$, and $\approx 220 \mathrm{~km}$, respectively) and the separation between all the sub-element strands also increases along the loop segment analyzed. Thus, the expansion observed in the open fan structures B and E are the result of sub-element strands both expanding and separating from each other simultaneously.

\subsection{Intensity versus Width}

Following the work of Klimchuk \& DeForest (2020), this section investigates the relationship between the peak intensity and width of the selected loops, and for the first time, extends this analysis to their sub-element strands. As is discussed in Klimchuk \& DeForest (2020), a negative correlation between 

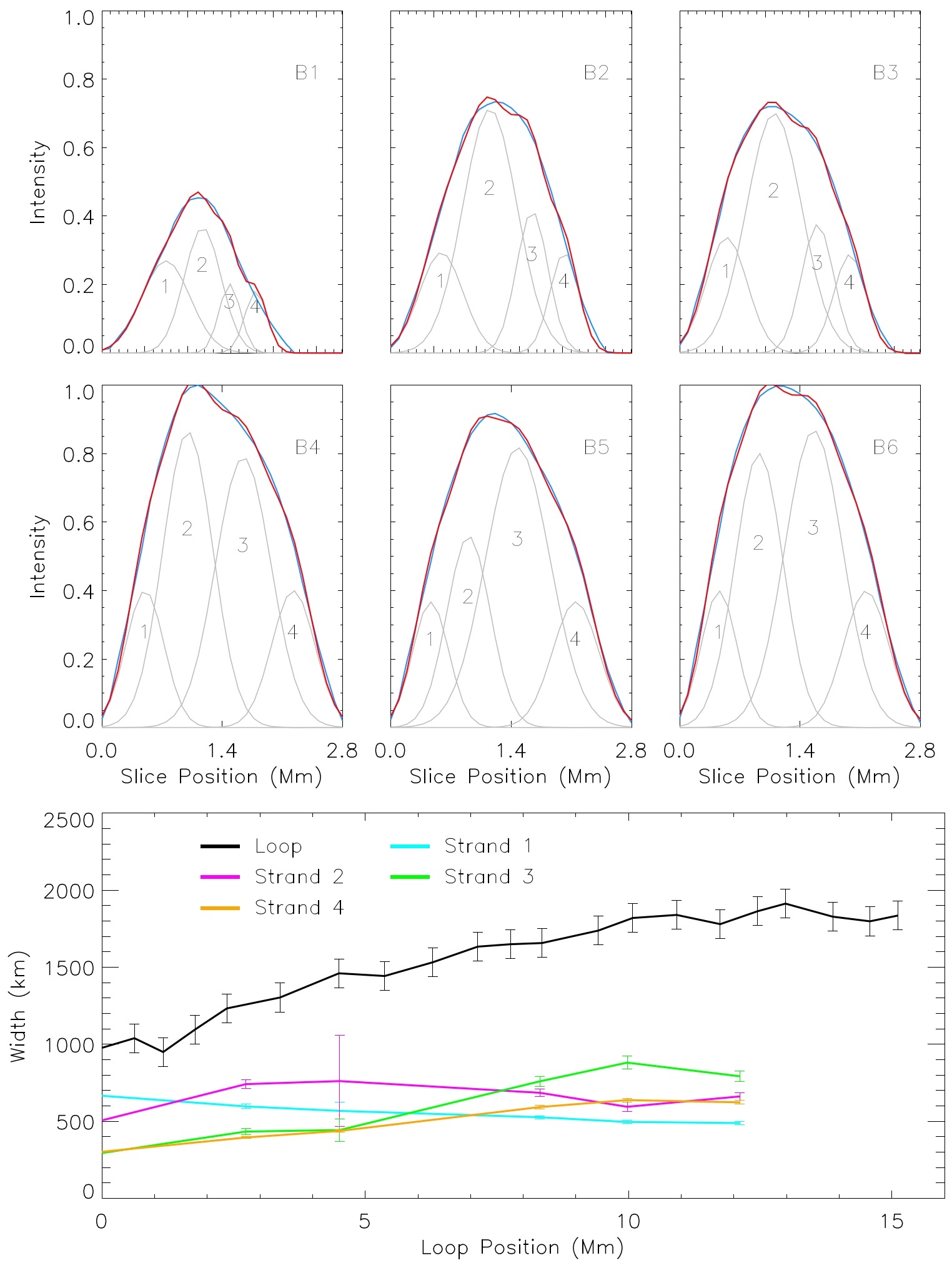

Figure 2. Panels B1-B6 show the normalized Hi-C 2.1 intensities (blue), the AICc determined fit (red), and the Gaussian profiles (gray) generated to produce the fit for loop B. Emission is normalized with respect to the maximum peak intensity of panels B1-B6. The Gaussian profiles are numbered in accordance with the bottom panel which shows the loop (black) and strand (cyan, magenta, green, and orange) widths as a function of distance along the segment of the loop analyzed. The error bars for the loop FWHM are \pm 1 Hi-C 2.1 pixel whereas the strand FWHM error bars are $5 \times$ the $1 \sigma$ uncertainty returned from the curve fitting method. Note that Loop Position $0-15 \mathrm{Mm}$ corresponds to a south-to-north orientation in Figure 1.

width and intensity may indicate that a loop has an elliptical cross section, whereas if there is no or positive correlation, the cross section is likely to be roughly circular.
Figure 5 top (bottom) shows the peak intensity of the loops (strands) as a function of FWHM. From visual inspection it can be seen that the peak intensities of loops B, C, and E 

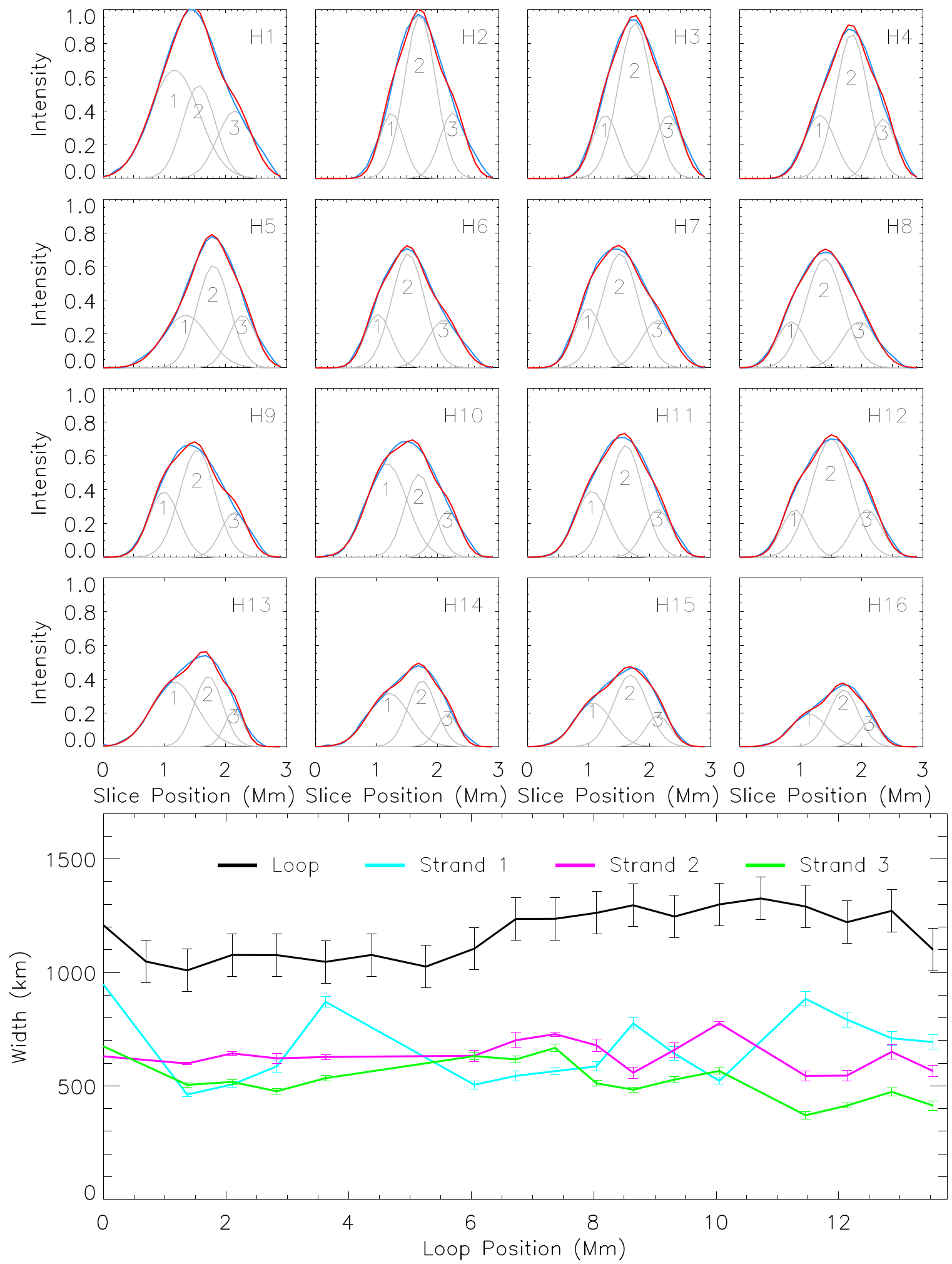

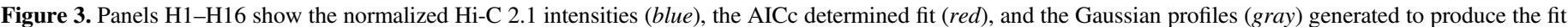

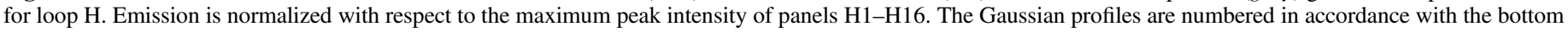

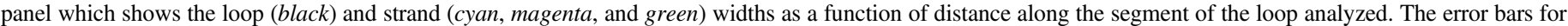

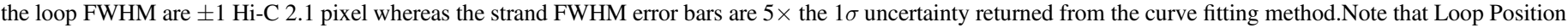
$0-13 \mathrm{Mm}$ corresponds to south-to-north orientation in Figure 1.

appear strongly correlated to the widths of the structures, while the remaining loops have weaker correlations where it is not possible to accurately determine whether the structures are positively or negatively correlated. As for the peak intensity of all the strands, they appear to predominantly be positively correlated to their widths-albeit to varying degrees with the strands of loop B showcasing the strongest correlation. 

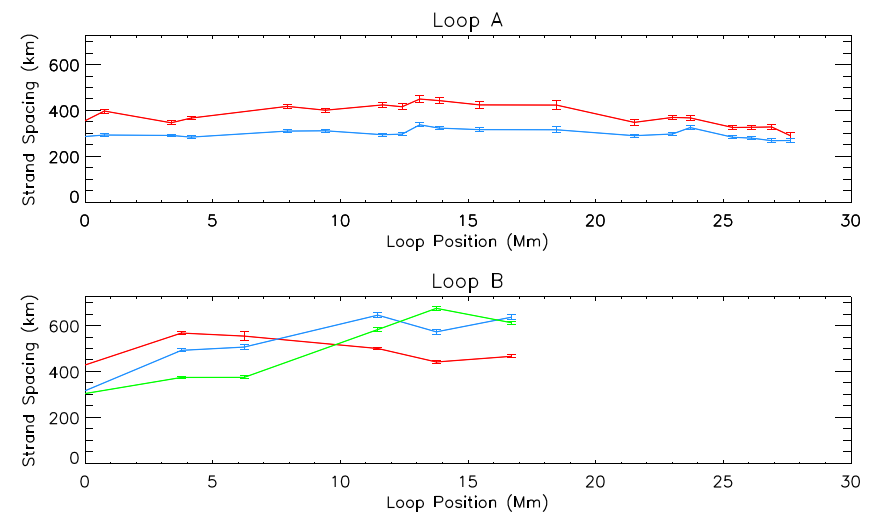

Loop C

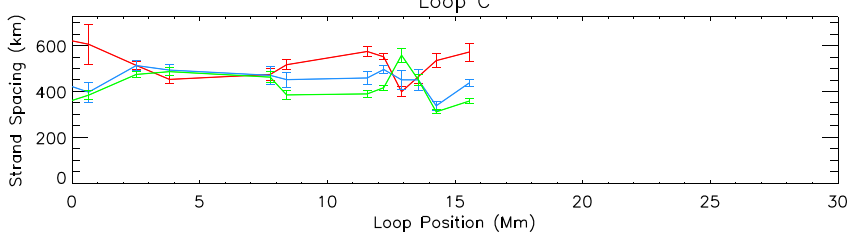

Loop D

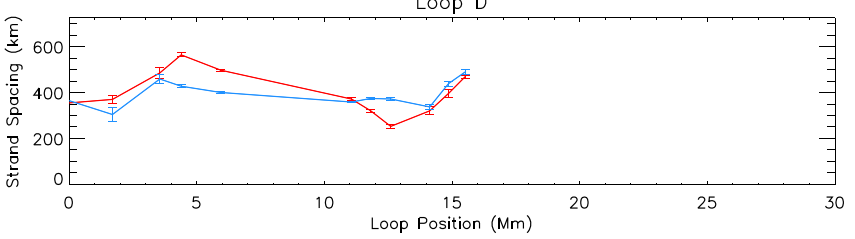

Loop E

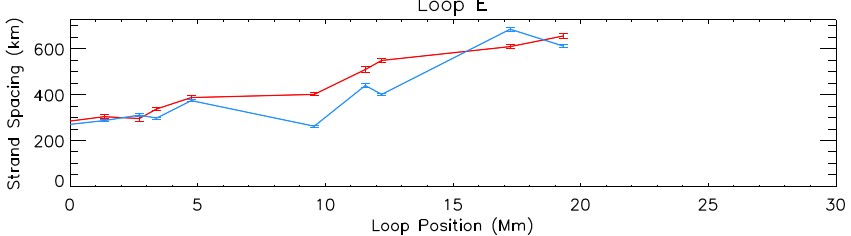

Loop F

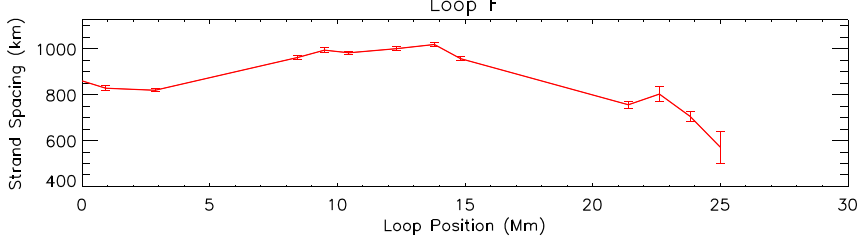

Loop G

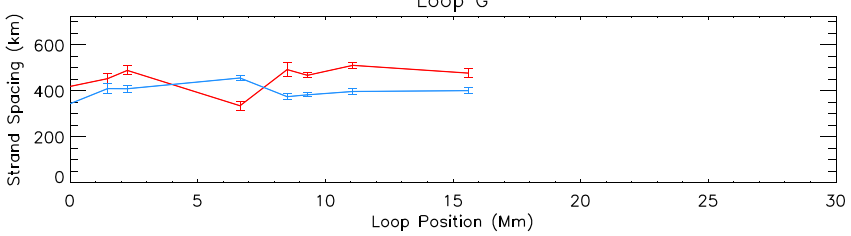

Loop $\mathrm{H}$

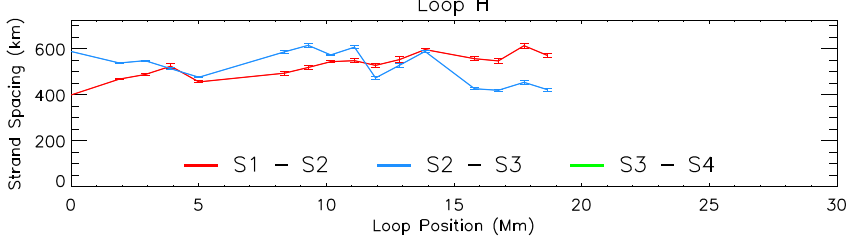

Figure 4. Figure shows the separation between the peak intensity of strands as a function of distance along the loop segments analyzed for strands 1 and 2 (blue), strands 2 and 3 (red), and strands 3 and 4 (green) for the eight loops investigated in this study. Note that increasing Loop Position for loops A, B, C, D, and H (E, F, and G) corresponds to a south-to-north (north-to-south) orientation in Figure 1.

To quantify whether the peak intensity of cross-sectional slices along a segment of a loop/strand is positively or negatively correlated to the FWHM, the Pearson correlation coefficient is determined, the results of which are presented in Table 1. The Pearson correlation coefficient, $P$ is defined as

$$
P=\frac{\operatorname{cov}(\mathrm{FWHM}, I)}{\sigma_{\mathrm{FWHM}} \sigma_{I}},
$$

where $\operatorname{cov}(W, I)$ is the covariance of FWHM and intensity, while the denominator is the product of their standard deviations. If a structure has a positive (negative) Pearson correlation then as the width increases the intensity increases (decreases). The coefficient magnitude varies between -1 and 1 with values of $P \approx 0$ corresponding to uncorrelated data, while values of -1 and 1 correspond to perfectly correlated data.

From Table 1, it is found that all the peak intensities of the four open fan loops are positively correlated to their width, with loops B, C, and E being strongly correlated, while loop A displays weak-to-moderate positive correlation. The weaker correlation of loop A is likely due to the fact that the structure exhibits minimal variation in the peak intensity and its width along the segment analyzed, and so deducing a relationship between the two is more difficult. As for the sub-element strands within the four open fan structures, a total of three strands are negatively correlated (loop A, strands 1, 3 and loop $\mathrm{B}$, strand 1), with the remaining 11 all exhibiting strong positive Pearson correlation coefficients. This indicates that as the structures broaden, peak intensities increase for open fan loops and their sub-element strands.

As for the magnetically closed structures, three of the four loops (D, F, and G) are positively correlated, while loop H is negatively correlated. All four loops have modest Pearson correlation coefficient magnitudes that are weaker than all the open fan loops bar loop A. Peculiarly, all the Pearson coefficients bar one of the sub-element strands are positively correlated for the magnetically closed structures despite the fact that loop H is negatively correlated. The other strand (loop G, strand 3), while having a negative Pearson coefficient, the absolute magnitude is such that the strand exhibits no linear correlation and thus is considered to be uncorrelated.

Focusing on loop $\mathrm{H}$, the loop width broadens as one travels along loop position (Figure 3 bottom) while the peak intensity of the cross-sectional slices decreases (Figure 3, H1-H16). Thus, there should be a negative correlation between the intensity and width for the loop. If we now consider the subelement strands, such as strand 2 say, it can be seen that as the peak intensity fluctuates, the FWHM remains relatively constant along the analyzed segment while the spacing between strands 1 and 2 (2 and 3) increases (decreases) (Figure 4) as the loop expands, i.e., the strands spread out (overlap more) along the integrated line of sight. All these factors together may explain a weak positive Pearson correlation coefficient for the strands of loop $\mathrm{H}$ despite the loop being negatively correlated.

As is noted in Klimchuk \& DeForest (2020), if a loop or strand were to be noncircular (such as an oval) and twisted, there would be an inverse relationship between the intensity and width. That is, the loop would have a higher (lower) peak intensity at narrower (broader) loop widths if relatively constant density and temperature are assumed. The majority of our results for open fan and closed loops, along with Klimchuk \& DeForest (2020), and nine of the 13 structures analyzed by McCarthy et al. (2021) indicate that the relationship between intensity and width may be positively correlated and thus their cross sections are approximately circular. 
Table 1

Pearson Coefficients and Expansion Factors for all the Loops/Strands Analyzed

\begin{tabular}{|c|c|c|c|c|c|c|}
\hline Structure & $\begin{array}{l}\text { Loop } \\
\text { Type }\end{array}$ & $\begin{array}{c}\text { Pearson } \\
\text { Coefficient }\end{array}$ & $\begin{array}{c}\text { Initial } \\
\text { Width }(\mathrm{km})\end{array}$ & $\begin{array}{c}\text { Final } \\
\text { Width }(\mathrm{km})\end{array}$ & $\begin{array}{c}\text { Total } \\
\text { Expansion }\end{array}$ & $\begin{array}{l}\text { Expansion } \\
\text { Rate }\left(\mathrm{Mm}^{-1}\right)\end{array}$ \\
\hline Loop A & Open Fan & 0.198 & 899.3 & 754.9 & -0.161 & -0.005 \\
\hline Strand 1 & & -0.247 & 437.3 & 495.4 & 0.133 & 0.005 \\
\hline Strand 2 & & 0.715 & 375.5 & 281.8 & -0.249 & -0.008 \\
\hline Strand 3 & & -0.587 & 327.9 & 321.4 & -0.020 & -0.001 \\
\hline Loop B & Open Fan & 0.995 & 976.5 & 1835.0 & 0.879 & 0.042 \\
\hline Strand 1 & & -0.926 & 665.3 & 488.5 & -0.266 & -0.021 \\
\hline Strand 2 & & 0.764 & 505.7 & 661.0 & 0.307 & 0.021 \\
\hline Strand 3 & & 0.981 & 293.8 & 791.9 & 1.696 & 0.102 \\
\hline Strand 4 & & 0.949 & 301.9 & 622.8 & 1.063 & 0.084 \\
\hline Loop C & Open Fan & 0.772 & 1581.4 & 1314.1 & -0.169 & -0.169 \\
\hline Strand 1 & & 0.931 & 791.2 & 673.9 & -0.148 & -0.011 \\
\hline Strand 2 & & 0.733 & 790.5 & 690.2 & -0.127 & -0.008 \\
\hline Strand 3 & & 0.911 & 470.6 & 404.6 & -0.140 & -0.009 \\
\hline Strand 4 & & 0.538 & 490.5 & 387.7 & -0.210 & -0.014 \\
\hline Loop D & Closed & 0.314 & 784.7 & 768.6 & -0.021 & -0.001 \\
\hline Strand 1 & & 0.525 & 768.9 & 403.6 & -0.475 & -0.031 \\
\hline Strand 2 & & 0.105 & 447.2 & 568.4 & 0.271 & 0.018 \\
\hline Strand 3 & & 0.465 & 442.6 & 493.2 & 0.115 & 0.007 \\
\hline Loop E & Open Fan & 0.869 & 651.9 & 1631.1 & 1.502 & 0.068 \\
\hline Strand 1 & & 0.844 & 510.5 & 768.3 & 0.505 & 0.027 \\
\hline Strand 2 & & 0.947 & 286.3 & 749.7 & 1.618 & 0.073 \\
\hline Strand 3 & & 0.789 & 297.1 & 513.6 & 0.729 & 0.038 \\
\hline Loop F & Closed & 0.384 & 1664.0 & 1199.8 & -0.279 & -0.010 \\
\hline Strand 1 & & 0.016 & 1096.0 & 1091.1 & -0.004 & $<-0.001$ \\
\hline Strand 2 & & 0.464 & 745.3 & 801.5 & 0.075 & 0.003 \\
\hline Loop G & Closed & 0.312 & 1054.4 & 1096.2 & 0.040 & 0.003 \\
\hline Strand 1 & & 0.143 & 694.8 & 651.1 & -0.063 & -0.004 \\
\hline Strand 2 & & 0.490 & 434.7 & 447.9 & 0.030 & 0.002 \\
\hline Strand 3 & & -0.053 & 429.4 & 359.7 & -0.162 & -0.010 \\
\hline Loop H & Closed & -0.437 & 1208.5 & 1100.5 & 0.052 & 0.003 \\
\hline Strand 1 & & 0.388 & 947.7 & 693.3 & -0.268 & -0.014 \\
\hline Strand 2 & & 0.395 & 630.5 & 565.5 & -0.103 & -0.006 \\
\hline Strand 3 & & 0.520 & 674.6 & 413.6 & -0.387 & -0.207 \\
\hline
\end{tabular}

However, it is worth noting that one magnetically closed loop, and three open fan strands of this study along with four loops analyzed by McCarthy et al. (2021) do support the idea that some loops and sub-element strands may be noncircular.

\section{Concluding Remarks}

Employing the analysis methods of Williams et al. (2020a, 2020b), this article investigates the relationship between width and peak intensity of eight relatively isolated coronal loops and extends this for the first time to sub-element strands within the Hi-C 2.1 data. These loops and strands are traced along segments $10-30 \mathrm{Mm}$ in length, which are of comparable length to the loops analyzed with the data from the first Hi-C mission (Klimchuk \& DeForest 2020) but are some 20-50 Mm shorter than those in the recent study by McCarthy et al. (2021).

The population of the eight loops analyzed is evenly split between open fan (loops A, B, C, and E) and magnetically closed (loops D, F, G, and H) structures and are thus treated independently from each other in our width expansion analysis. The segments analyzed for loops $\mathrm{B}$ and $\mathrm{E}$ reside near the visible footpoints (Figure 1) of the open fan structures and thus undergo significant expansion whereby their widths increase an additional $80 \%$ and $150 \%$ (see Total Expansion, Table 1) of their initial value. Conversely, the segments analyzed for loops $A$ and $C$ (Figures 6 and 7) show minimal width variation along their length. These results suggest that open fan loops may expand rapidly near their footpoints and after which their crosssectional width may remain relatively constant. With that said, a more comprehensive data set is required from future highresolution missions to confirm this as current and archival data lack the resolving power to accurately quantify the expansion seen with Hi-C 2.1.

As with previous loop width studies (Klimchuk et al. 1992; Klimchuk 2000, 2015; López Fuentes \& Klimchuk 2006), the magnetically closed structures analyzed in this article exhibit little expansion with three of the four loops showing $<6 \%$ (108 km; <1.2 Hi-C 2.1 pixels) expansion (Table 1) along the analyzed segments. Similarly, the width and separation distance of the sub-element strands of these loops remain largely constant along the loop portions under consideration in this study. One caveat to this is that loop $\mathrm{D}$ can be seen to expand and contract periodically. 


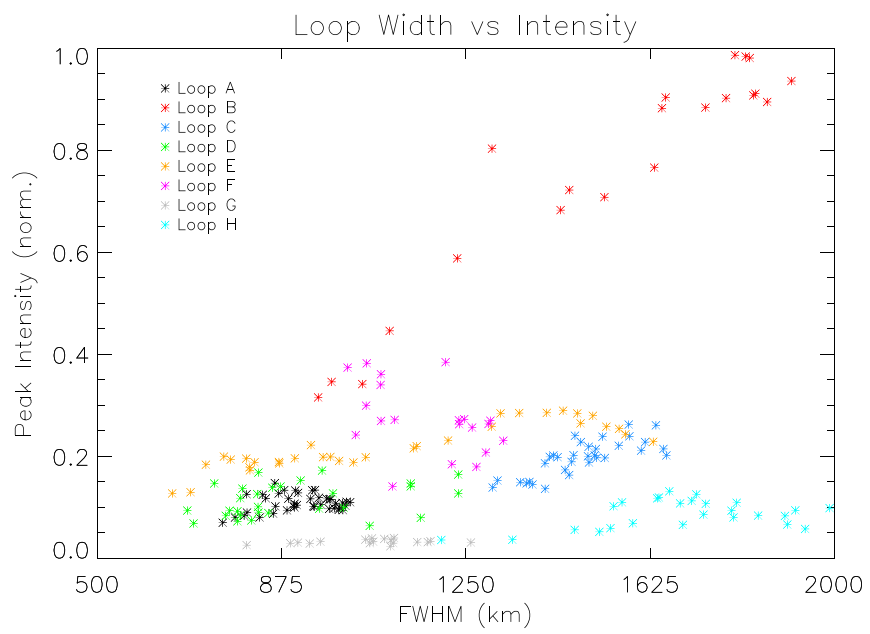

Strand Width vs Intensity

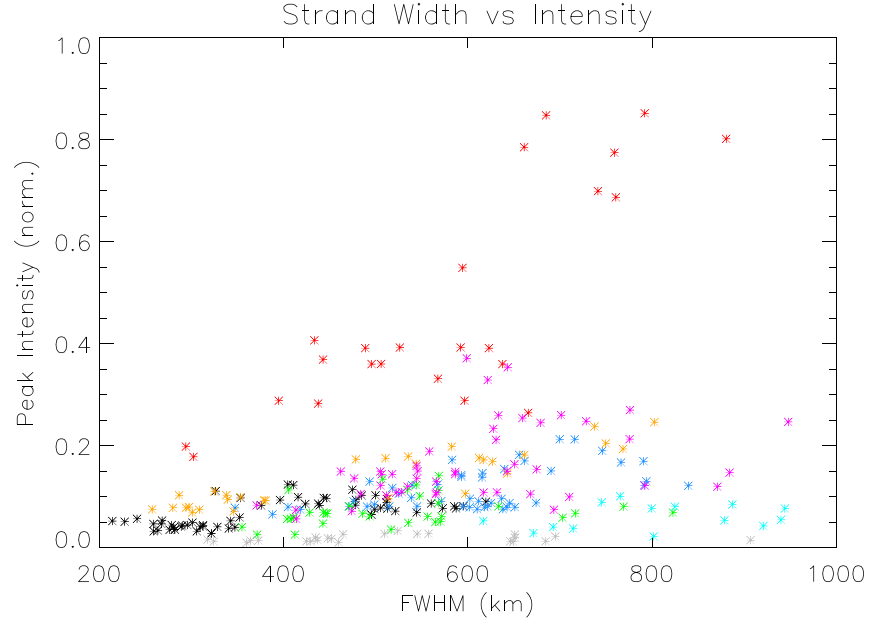

Figure 5. Top: Peak intensities are plotted against their widths for loops A-H. Bottom: Peak intensities of the strands contained within loops A-H are plotted against their widths. No distinction is made here about individual strands but rather the strands as a collective for each loop, i.e., strands 1-3 of loop A are all plotted as black asterisks, strands 1-3 of loop B are plotted as red asterisks, and strands 1-4 of loop $\mathrm{C}$ are plotted as blue asterisks, etc.

As discussed by Klimchuk \& DeForest (2020), the largest error in coronal loop width analysis is a direct result of performing background subtraction due to the relative magnitudes of the loop and line-of-sight emission that is removed. Close inspection of the FOV image (Figure 1) reveals multiple structures intersecting/crossing loop D, which makes it more difficult to employ a consistent background removal along the loop segment. As such, these variations in loop width are likely to be the caused by background subtraction due to the crossing of multiple structures along the line of sight rather than because the loop is a twisted structure of noncircular cross section (Malanushenko \& Schrijver 2013).

Generalizing the data set as a whole, the expansion of loops can be quantified by the sub-element strands broadening and separating from each other simultaneously along the segments considered, which leads to the increasing cross-sectional loop widths seen in loops B and E. Similarly, the lack of expansion seen in the other six structures is caused by strands whose widths and separation remain relatively constant along the loop segments analyzed.

Upon exploring the relationship between loop width and peak intensity, this article finds that seven of the eight loops analyzed $(\approx 88 \%)$ are positively correlated, with the other loop being negatively correlated. These results match previous findings (West et al. 2014; Kucera et al. 2019; Klimchuk \& DeForest 2020) with Klimchuk \& DeForest (2020) being a notable comparison due to their work also examining loops of similar lengths to the ones in this study with $\mathrm{Hi}-\mathrm{C}$, albeit with the $193 \AA$ data set. As such, our results support the Klimchuk \& DeForest (2020) hypothesis that loops are more likely to exhibit circular cross sections rather than elliptical ones (Malanushenko \& Schrijver 2013; McCarthy et al. 2021), which would be the case if the peak intensities and widths of the cross sectional slices were negatively correlated. As for the coronal strands, we obtain a similar outcome with 20 strands $(80 \%)$ showing positive correlation, three strands (12\%) showing negative correlation and two strands (8\%) being uncorrelated.

The findings presented in this article for magnetically closed coronal loops and their sub-element strands support the discussion of Klimchuk (2000) and Williams et al. (2020a, 2020b) in regards to coronal heating. That is, whatever mechanism is responsible for the coronal heating, theoretical models may need to reproduce monolithic strands whose cross sections (i) have widths at the same order of an AIA pixel $(\approx 435 \mathrm{~km})$, and (ii) are approximately circular along their length. These implications for coronal heating are contingent on coronal loops being compact structures that are subjected to twist. In particular, fitting multiple nearly cylindrical Gaussianshaped strands relies heavily on the assumption that curvilinear features in the Hi-C 2.1 image plane do indeed correspond to compact structures in three dimensions. While this assumption is plausible, other explanations may be equally valid in explaining the cross-sectional profiles observed. As is discussed in McCarthy et al. (2021), monolithic loops when viewed from a single vantage point - such as AIA or in this study Hi-C 2.1-may in fact be loops with non-fixed aspect ratios and/or exhibit anisotropic expansion. They may also be the result of projection effects of complicated bright manifolds in the corona, or some other complex density enhancement. Thus, further three-dimensional analysis is required to confirm whether coronal loops are possibly well represented by a collection of sub-element strands.

As with the West et al. (2014) (11 loops), Klimchuk \& DeForest (2020) (20 loops), and McCarthy et al. (2021) (13 loops) studies, this analysis is on a small sample size-largely due to the difficulty in tracing isolated segments of coronal loops within the Hi-C 2.1 data-and as suggested by Malanushenko \& Schrijver (2013) may be subject to selection bias. Further high-resolution data is required, such as from Solar Orbiter's Extreme Ultraviolet Imager $\left(\approx 0.1^{\prime \prime} \mathrm{pixel}^{-1}\right.$ at $0.28 \mathrm{au}$ ) instrument, to explore the relationship between intensity and width in the hope of understanding the fundamental geometry of these important structures in the quest for solving the coronal heating problem.

T.W. and H.M. gratefully acknowledge support by Leverhulme grant RPG-2019-361. H.M. also acknowledges STFC grant ST/S000518/1.

\section{Appendix \\ Fitting Coronal Loop Sub-elements}

In this appendix, the additional figures discussed in Section 3 are presented. In Figure 6, the top panels (A1-A20) show the normalized Hi-C 2.1 intensity slice (blue) and the Gaussian 

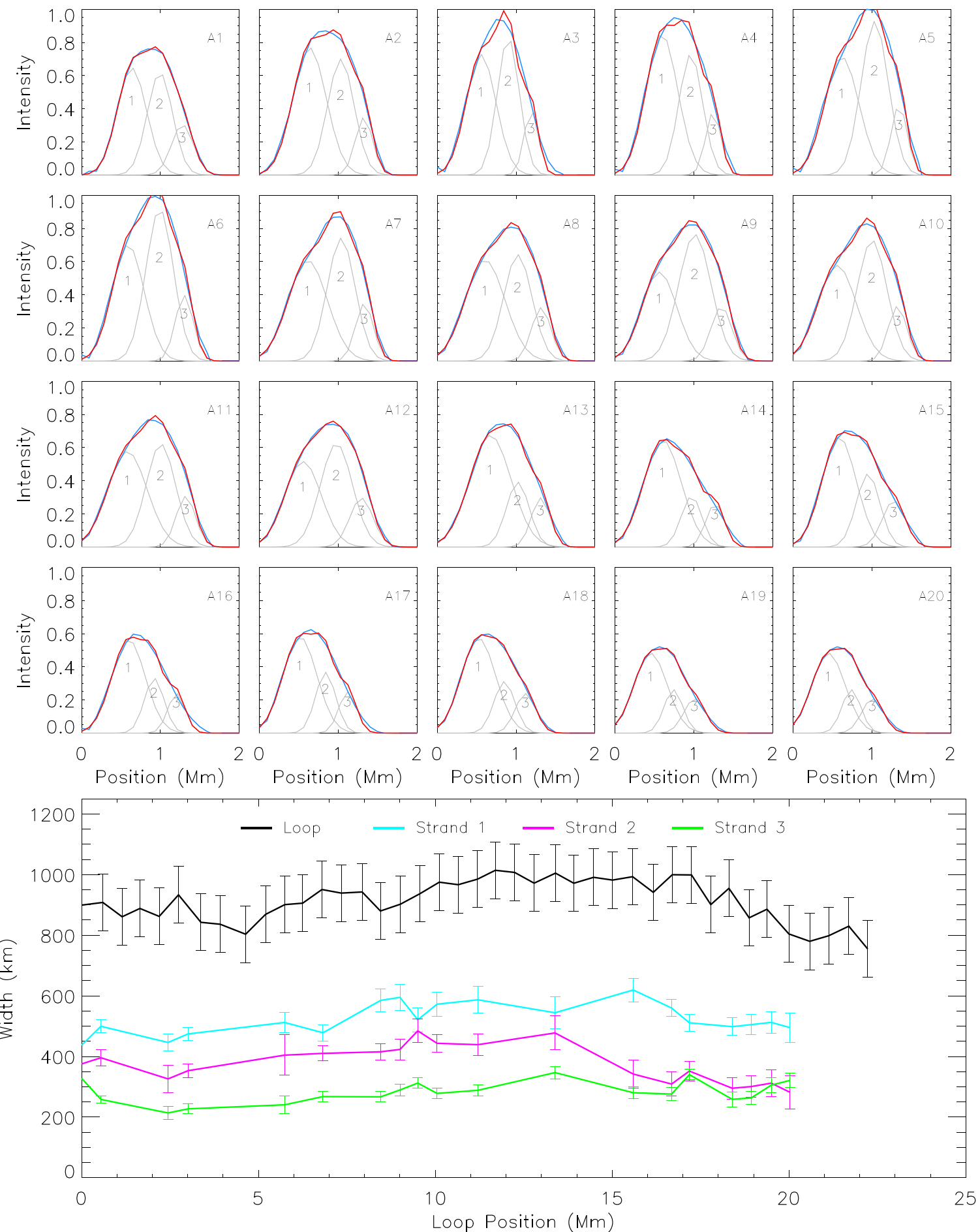

Figure 6. Panels A1-A20 show the normalized Hi-C 2.1 intensities (blue), the AICc determined fit (red), and the Gaussian profiles (gray) generated to produce the fit for loop A. Emission is normalized with respect to the maximum peak intensity of panels A1-A20. The Gaussian profiles are numbered in accordance with the bottom panel, which shows the loop (black) and strand (cyan, magenta, and green) widths as a function of distance along the segment of the loop analyzed. The error bars for the loop FWHM are \pm 1 Hi-C 2.1 pixel whereas the strand FWHM error bars are $5 \times$ the $1 \sigma$ uncertainty returned from the curve fitting method. Note that loop position $0-25 \mathrm{Mm}$ corresponds to a south-to-north orientation in Figure 1.

profiles (gray) that are used to generate the fit (red). The crosssectional profiles are each normalized to the maximum intensity of the loop segment. The bottom panel shows the cross-sectional width of the loop (black) and the sub-element strands (cyan, magenta, green) as a function of position along the loop segment. The error bars for the loop widths indicate $\pm 1 \mathrm{Hi}-\mathrm{C} 2.1$ pixel while the error bars of the sub- element strand widths are the $1 \sigma$ errors returned from the curve fitting method. The same plots are shown for loops $\mathrm{C}$ (Figure 7), D (Figure 8), E (Figure 9), F (Figure 10), and G (Figure 11). As can be seen from these figures, magnetically closed loops, D, F, and G, and open fan loops A and C show little variance in the cross-sectional loop width versus length, while open fan loop E undergoes significant expansion. 

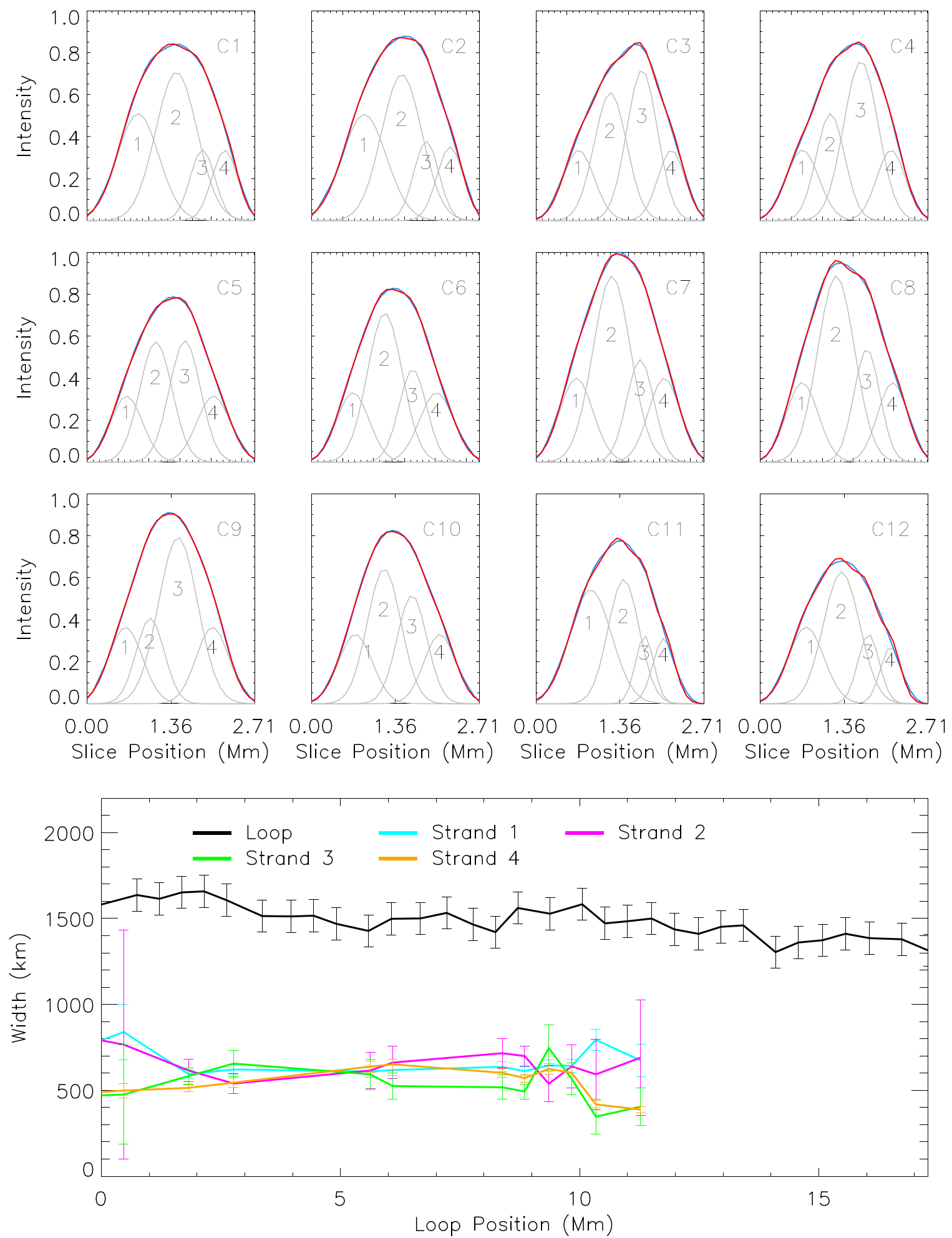

Figure 7. Panels $\mathrm{C} 1-\mathrm{C} 12$ show the normalized Hi-C 2.1 intensities (blue), the AICc determined fit (red), and the Gaussian profiles (gray) generated to produce the fit for loop C. Emission is normalized with respect to the maximum peak intensity of panels $\mathrm{C} 1-\mathrm{C} 12$. The Gaussian profiles are numbered in accordance with the bottom panel, which shows the loop (black) and strand (cyan, magenta, green, and orange) widths as a function of distance along the segment of the loop analyzed. The error bars for the loop FWHM are \pm 1 Hi-C 2.1 pixel whereas the strand FWHM error bars are $5 \times$ the $1 \sigma$ uncertainty returned from the curve fitting method. Note that loop position $0-17 \mathrm{Mm}$ corresponds to a south-to-north orientation in Figure 1. 

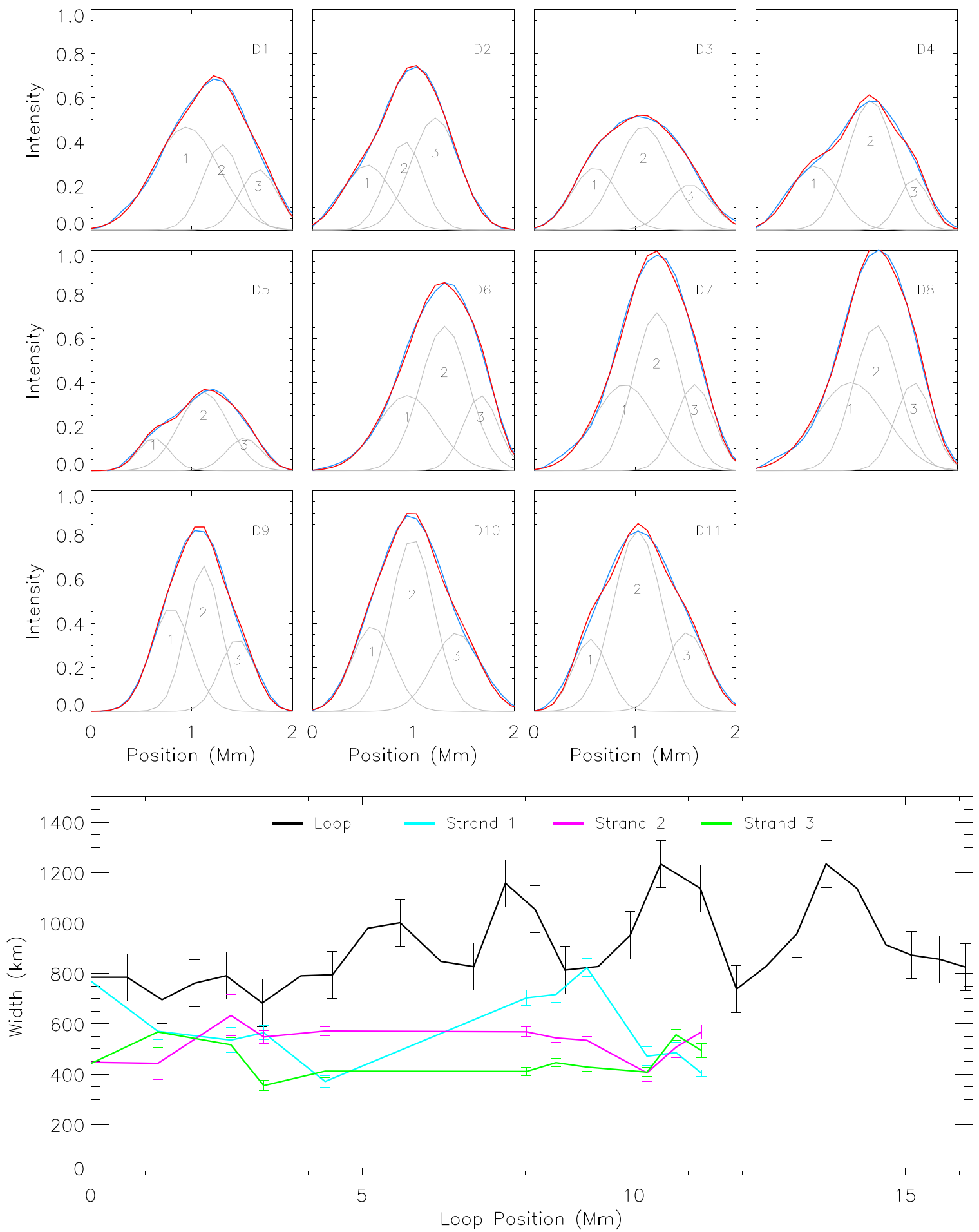

Figure 8. Panels D1-D11 show the normalized Hi-C 2.1 intensities (blue), the AICc determined fit (red), and the Gaussian profiles (gray) generated to produce the fit for loop D. Emission is normalized with respect to the maximum peak intensity of panels D1-D11. The Gaussian profiles are numbered in accordance with the bottom panel, which shows the loop (black) and strand (cyan, magenta, and green) widths as a function of distance along the segment of the loop analyzed. The error bars for the loop FWHM are \pm 1 Hi-C 2.1 pixel, whereas the strand FWHM error bars are $5 \times$ the $1 \sigma$ uncertainty returned from the curve fitting method. Note that loop position $0-16 \mathrm{Mm}$ corresponds to a south-to-north orientation in Figure 1. 

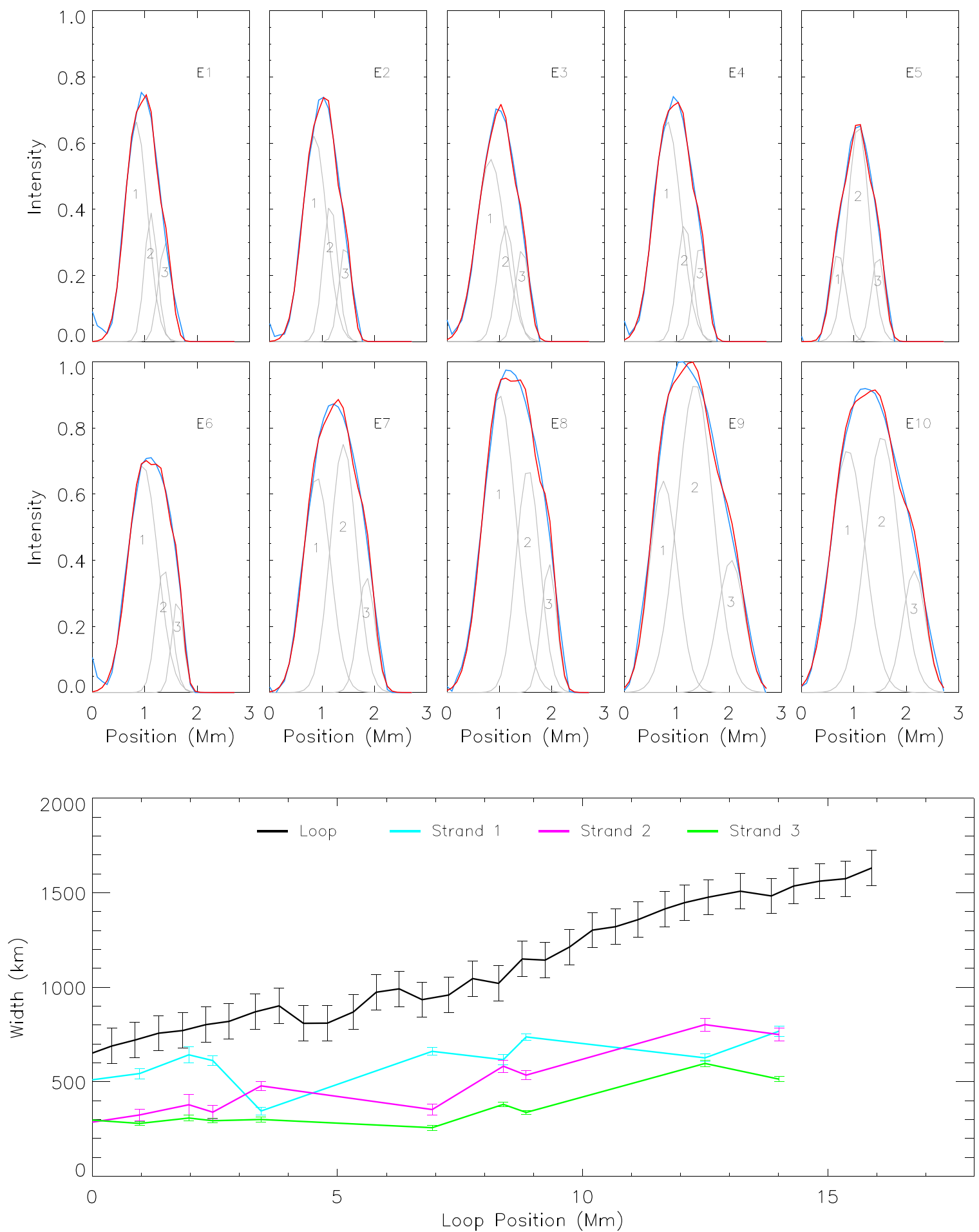

Figure 9. Panels E1-E10 show the normalized Hi-C 2.1 intensities (blue), the AICc determined fit (red), and the Gaussian profiles (gray) generated to produce the fit for loop E. Emission is normalized with respect to the maximum peak intensity of panels E1-E10. The Gaussian profiles are numbered in accordance with the bottom panel, which shows the loop (black) and strand (cyan, magenta, and green) widths as a function of distance along the segment of the loop analyzed. The error bars for the loop FWHM are \pm 1 Hi-C 2.1 pixel, whereas the strand FWHM error bars are $5 \times$ the $1 \sigma$ uncertainty returned from the curve fitting method. Note that loop position $0-18 \mathrm{Mm}$ corresponds to a north-to-south orientation in Figure 1. 

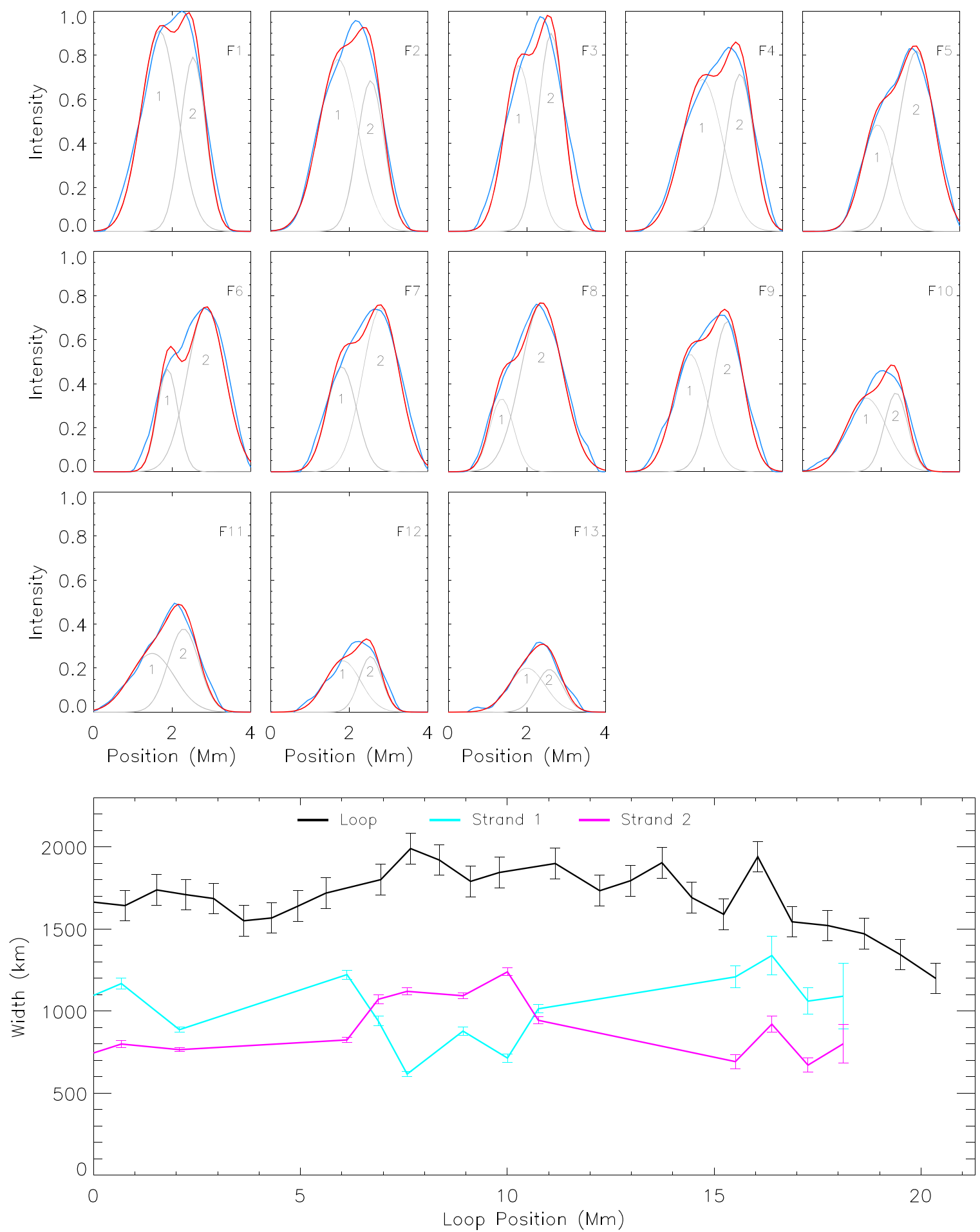

Figure 10. Panels F1-F13 show the normalized Hi-C 2.1 intensities (blue), the AICc determined fit (red), and the Gaussian profiles (gray) generated to produce the fit for loop F. Emission is normalized with respect to the maximum peak intensity of panels F1-F13. The Gaussian profiles are numbered in accordance with the bottom panel, which shows the loop (black) and strand (cyan, magenta, and green) widths as a function of distance along the segment of the loop analyzed. The error bars for the loop FWHM are \pm 1 Hi-C 2.1 pixel, whereas the strand FWHM error bars are $5 \times$ the $1 \sigma$ uncertainty returned from the curve fitting method. Note that loop position 0-21 Mm corresponds to a north-to-south orientation in Figure 1. 

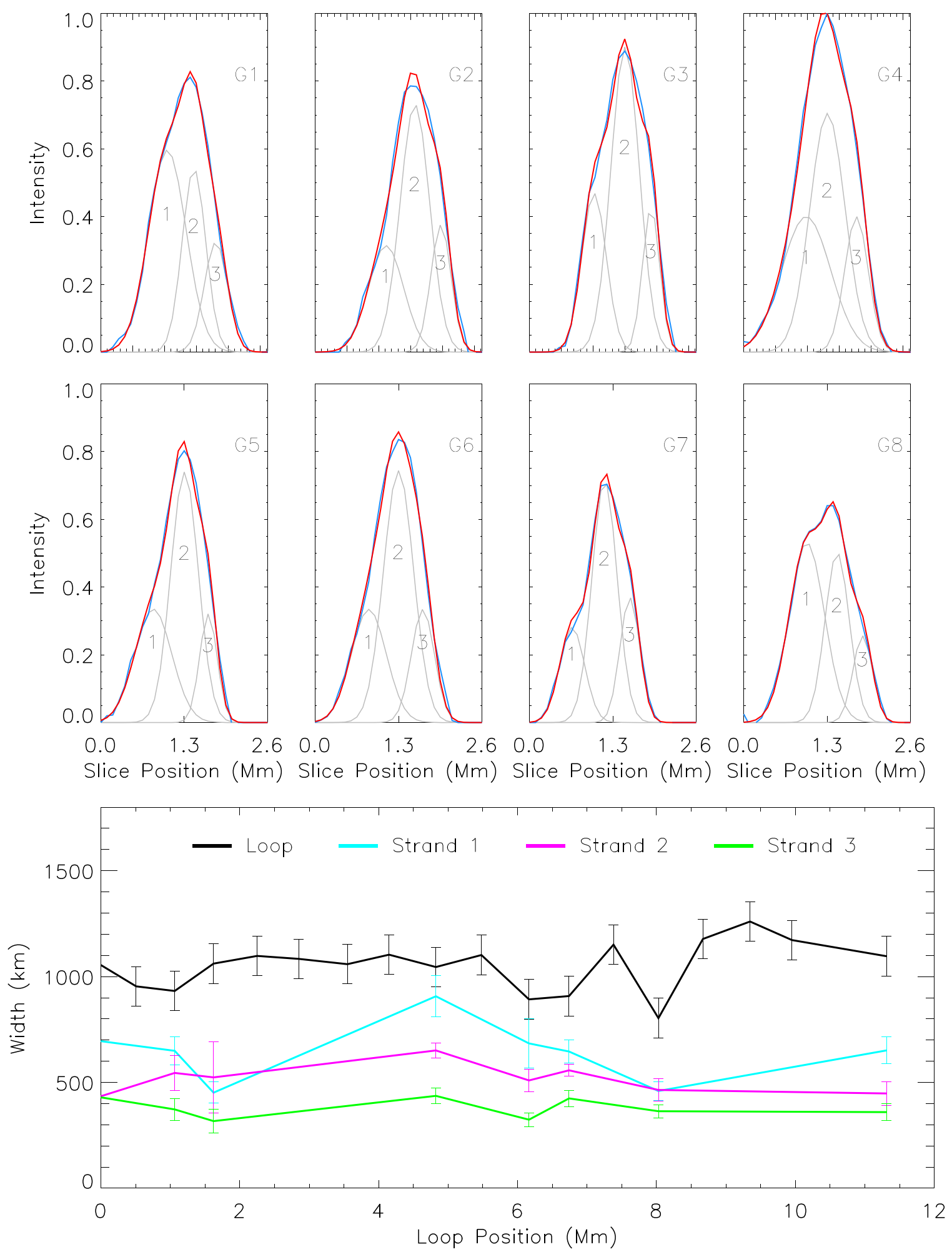

Figure 11. Panels G1-G11 show the normalized Hi-C 2.1 intensities (blue), the AICc determined fit (red), and the Gaussian profiles (gray) generated to produce the fit for loop E. The Gaussian profiles are numbered in accordance with the bottom panel, which shows the loop (black) and strand (cyan, magenta, and green) widths as a function of distance along the segment of the loop analyzed. The error bars for the loop FWHM are \pm 1 Hi-C 2.1 pixel, whereas the strand FWHM error bars are $5 \times$ the $1 \sigma$ uncertainty returned from the curve fitting method. Note that loop position $0-12 \mathrm{Mm}$ corresponds to a north-to-south orientation in Figure 1 . 


\section{ORCID iDs}

Thomas Williams @i https://orcid.org/0000-0002-2006-6096

Robert W. Walsh (1) https://orcid.org/0000-0002-1025-9863

Huw Morgan (ib https://orcid.org/0000-0002-6547-5838

\section{References}

Akaike, H. 1974, ITAC, 19, 716

Aschwanden, M. J., \& Schrijver, C. J. 2011, ApJ, 736, 102

Brooks, D. H., Reep, J. W., \& Warren, H. P. 2016, ApJL, 826, L18

Brooks, D. H., Warren, H. P., Ugarte-Urra, I., \& Winebarger, A. R. 2013, ApJL, 772, L19

Cirtain, J. W., Golub, L., Winebarger, A. R., et al. 2013, Natur, 493, 501

Howard, R. A., Moses, J., Vourlidas, J. S., et al. 2008, SSRv, 136, 67

Kobayashi, K., Cirtain, J., Winebarger, A. R., et al. 2014, SoPh, 289, 4393

Klimchuk, J. A. 2000, SoPh, 193, 53

Klimchuk, J. A. 2015, RSPTA, 373, 20140256

Klimchuk, J. A., Antiochos, S. K., \& Norton, D. 2000, ApJ, 542, 504

Klimchuk, J. A., \& DeForest, C. E. 2020, ApJ, 900, 167

Klimchuk, J. A., Lemen, J. R., Feldmen, U., Tsuneta, S., \& Uchida, Y. 1992, PASJ, 44, L181

Kucera, T. A., Young, P. R., Klimchuk, J. A., \& DeForest, C. E. 2019, ApJ, 885, 7
Lemen, J. R., Title, A. M., Akin, D. J., et al. 2012, SoPh, 275, 17

López Fuentes, M. C., \& Klimchuk, J. A. 2006, ApJ, 639, 459

Malanushenko, A., \& Schrijver, C. J. 2013, ApJ, 775, 120

Markwardt, C. B. 2009, in ADASS, ASP Conf. Ser., 411, ed. D. Bohlender, P. Dowler, \& D. Durand (San Francisco, CA: Astronomical Society of the Pacific), 251

McCarthy, M. I., Longcope, D. W., \& Malanushenko, A. 2021, ApJ, In Press McClymont, Z., \& Mikic, Z. 1994, ApJ, 422, 899

Moré, J. J. 1978, in Numerical Analysis (Lecture Notes in Mathematics) ed. G. A. Watson, 630 (Berlin: Springer)

Morgan, H., \& Druckmüller, M. 2014, SoPh, 289, 2945

Morton, R. J., \& McLaughlin, J. A. 2013, A\&A, 553, L10

Parker, E. N. 1983, ApJ, 264, 642

Parker, E. N. 1988, ApJ, 330, 474

Peter, H., Bingert, S., Klimchuk, J. A., et al. 2013, A\&A, 556, A104

Pontin, D. I., Janvier, M., Tiwari, S. K., et al. 2017, ApJ, 837, 108

Rachmeler, A. L., Winebarger, A. R., Savage, A. L., et al. 2019, SoPh, 294, 174

Watko, J., \& Klimchuk, J. A. 2000, SoPh, 193, 77

West, M., Zhukov, A., \& Klimchuk, J. A. 2014, in 40th COSPAR Scientific Assembly, Cross-Sectional Properties of Coronal Loops, Vol. E2

Williams, T., Walsh, R. W., Peter, H., et al. 2020b, ApJ, 902, 90

Williams, T., Walsh, R. W., Winebarger, A. R., et al. 2020a, ApJ, 892, 134

Yi, L., Liu, Z., Wang, K., et al. 2015, NIMPA, 775, 12 